\title{
ESTUDIO DE ESTEREOTIPO Y METAESTEREOTIPO DE UNA MUESTRA DE LA POBLACIÓN CHILOTA RESIDENTE EN LA CIUDAD DE PUNTA ARENAS
}

STEREOTYPE AND META-STEREOTYPE STUDY OF A SAMPLE CHILOTA POPULATION LIVING IN PUNTA ARENAS CITY

PAULINA ALARCÓN PAZ ${ }_{1}$; CLAUDIA ESTRADA GOIC 2 (UNIVERSIDAD DE MAGALLANES, Punta Arenas, Chile).

\begin{abstract}
RESUMEN
La presente investigación tuvo como propósito conocer los principales adjetivos que configuran el autoestereotipo y el metaestereotipo de los chilotes residentes en Magallanes, así como también busca conocer el nivel de identificación que manifiestan éstos con su endogrupo. "Chilotes" es un término utilizado para designar al grupo de inmigrantes desde la Isla de Chiloé (Décima Región) a otra comunidad o Región. Se adaptó la Técnica de generación de adjetivos y la Técnica de lista de adjetivos de Katz y Braly (1933), para medir los estereotipos, mientras que la identificación fue medida con una escala ad-hoc. La muestra no probabilística de tipo intencional, estuvo conformada por 65 personas oriundas de Chiloé y/0 descendientes de chilotes que actualmente viven en la comuna de Punta Arenas. Los resultados indican que los adjetivos asignados al autoestereotipo y metaestereotipo son en su mayoría de tendencia positiva aún cuando los encuestados tienen la clara percepción de que el exogrupo (magallánicos no chilotes) no los evalúa tan favorablemente. Sumado a eso, los chilotes presentan un alto nivel de identificación con su endogrupo. Dichos hallazgos son discutidos en el marco de las relaciones intergrupales y teoría de la identidad social.
\end{abstract}

PALABRAS CLAVE: Auto-estereotipo; metaestereotipo; identidad; chilotes.

\section{ABSTRACT}

The present investigation had as a purpose to know the main adjectives that form the self-stereotype and the meta-stereotype of the "chilotes" that reside in Magallanes, as well as it also seeks to know the level of identification that these demonstrate with their ingroup. "Chilotes" is a term used to designate the group of immigrants which came from Chiloé Island (Región de los Lagos, Chile) to another community or region. In order to measure the stereotypes, there were adapted both: the technique of "Generation of adjectives" and the technique of "List of adjectives" by Katz \& Braly (1933), whereas the identification was measured with an "adhoc" scale. The not probabilistic sample type was formed by 65 native people from Chiloé and/or descendants of "chilotes" that currently live in Punta Arenas. The results indicate that the adjectives assigned to the selfstereotype and meta-stereotype are mainly of positive tendency even though the polled ones have the clear perception that the outgroup (magellan people that are not "chilotes") do not evaluate them in a completely favourable way. In addition, the "chilotes" show a high level of identification with their ingroup. The above findings are discussed within the framework of intergroup relations and social identity theory.

KEY WORDS: Self-stereotype; meta-stereotype; identity; chilotes.

1. Magister en Ciencias Sociales de la Universidad de Magallanes.

2. Doctora y académica afiliada a la Universidad de Magallanes.

Correspondencia: Claudia Estrada Goic: Escuela de Psicología, Universidad de Magallanes, Av. Bulnes 01855 - (61) 207 - 944.6200000 Punta Arenas, Chile. E-mail: claudia.estrada@umag.cl 


\section{MARCO TEÓRICO}

Actualmente nuestra sociedad experimenta cambios acelerados a nivel económico, político, social y tecnológico. En palabras de Sandoval (1998), una serie de "mutaciones culturales" han influido notoriamente en la configuración de identidad de los diversos actores que participan activamente en estos procesos.

Caracterizada por la belleza de sus paisajes y sus tradiciones ancestrales, la provincia de Chiloé, ubicada en la nueva región de los Lagos, Chile, posee un total de 154.766 habitantes (Censo 2002), que descienden en su gran mayoría de la mezcla entre indígenas y españoles; no obstante, a raíz de las corrientes migratorias que se han dado en nuestro país (780.000 según censo 2002) un porcentaje importante de la población chilota ha debido trasladarse del campo a la ciudad o a una región ajena a la suya en busca de oportunidades de trabajo, capacitación profesional y otros, enfrentándose así a nuevas variables contextuales que amenazan su especificidad y generan dilemas en su identidad social.

En el caso de la ciudad de Punta Arenas, los chilotes han ejercido un rol sumamente importante, principalmente como emigrantes y fundadores de los territorios australes desde el año 1843, cuando durante el gobierno de Manuel Bulnes se concretaron los planes de incorporar las tierras patagónicas a la soberanía de Chile (Martinic, 2002).

Numerosos han sido los aportes de la población chilota a la región de Magallanes, partiendo por su progresiva presencia en estas tierras. Actualmente no existen estadísticas de la población total de chilotes que residen en la región, así como tampoco hay tabulaciones específicas que registren las corrientes migratorias desde Chiloé hacia la región de Magallanes. No obstante, en este ultimo caso, es posible hacer una proyección a partir de las estadísticas vitales y migratorias de la Región de los Lagos correspondientes al censo del año 2002, que permite obtener una población potencial aproximada de 840 chilotes, de 5 años 0 más que emigran a la Patagonia. Aquel número no es menor si consideramos que en la actualidad la región de Magallanes se caracteriza por poseer una tasa de migración neta de -6,7 (Censo 2002) y dentro de su tasa de inmigración es la región de los Lagos quien aporta a Magallanes el mayor porcentaje de emigrantes.

En la actualidad, el pueblo chilote se ve enfrentado a un contexto social representado por una cultura magallánica; que según estudios preliminares (Estrada y Avendaño, 2008) posee ciertas características tales como la tendencia a ser estable, inamovible y dificultar la inserción de nuevos miembros. Esta variable contextual, puede contribuir a desarrollar una nueva identidad social basada en autoestereotipos y metaestereotipos que surgen a raíz de este encuentro entre grupos sociales, que definen el nivel de identificación con el endogrupo.

Esta temática resulta de particular interés por cuanto no existen antecedentes empíricos a nivel regional que aborden desde una postura social y psicológica la identidad de la población chilota residente en la comuna de Punta Arenas, y menos aún de elementos que forman parte de esa identidad, como es el caso de los estereotipos. De hecho, en nuestro país los estudios de metaestereotipo principalmente están vinculados a la población indígena mapuche (Saiz, Merino y Quilaqueo, 2009) siendo prácticamente nula la investigación que se ocupa del autoestereotipo y metaestereotipo de manera simultánea. En las Ciencias Sociales, la identidad cada vez es un tema más frecuente ya que sin duda es capaz de generar conciencia de la reflexividad humana, un claro sentido de pertenencia y una postura definida frente a la sociedad. Además, el estudio en este 
campo permite explicar potencialmente fenómenos que surgen a raíz de la interacción y que finalmente determinan como las personas, grupos y comunidades se vinculan, se relacionan dialécticamente y conciben lo social.

La identidad, al ser un concepto universal tiene diversos significados, por lo que se utiliza en variados contextos dependiendo de la tradición del pensamiento o disciplina a la cual se adhiera (Larraín, 2001 y Therborn, 2000). Desde el ámbito de la psicología, Sherman (en Banon y Byme, 2005) postula que la identidad social es la definición que cada uno hace de sí mismo a partir de los atributos personales y aquellos que comparte con grupos sociales. Lo anterior implica que al hablar de identidad, los aspectos individuales y colectivos (sociales), que rodean a una persona tienden a confluir y rara vez pueden prescindir uno del otro.

La teoría de la identidad social de Tajfel (1984), por su parte, plantea que el autoconcepto individual estaría conformado también por su identidad social, es decir, por la consciencia de pertenecer a determinados grupos sociales y el valor emocional que se le atribuye a dicha membresía.

Sin duda, la identidad es una necesidad básica del ser humano y toda persona se siente motivada a evaluarse a si mismo de manera positiva. En ese entendido, la pertenencia a ciertos grupos sociales, además de generar sesgo endogrupal, cumple un rol fundamental en el autoconcepto. Las personas pueden definirse y evaluarse a partir de su pertenencia a una categoría social, y buscan identificarse y permanecer en aquellos grupos que aportan valor positivo a su identidad (Tajfel y Turner, 1979). La identificación, vendría a ser el elemento necesario para determinar la existencia de un grupo, en cuanto al compartir una misma identidad social y percibirse como miembro de una misma categoría, se potencian las diferencias intergrupales y se fomenta la aparición de los estereotipos (Scandroglio, López y San José, 2008, Secord y Backman, 1976).

Los estereotipos interesan desde tempranamente a la psicología social (Lippman, 1922). Definidos como el conjunto de creencias, cualidades 0 suposiciones que se atribuyen a las categorías sociales, son una parte constituyente de la identidad social, por cuanto recogen tanto la información que otros atribuyen a los grupos a los que pertenecemos (Saiz, Rapiman y Mladinic, 2008), como también la que un grupo se atribuye a sí mismo (autoestereotipo) Pueden concebirse como un caso especial de categorización que acentúa las similitudes intra-grupales y las diferencias inter-grupales (Tajfel, 1984) y es a través del aprendizaje cultural y las vivencias personales que los estereotipos se desarrollarían (Worchel, Cooper, Goethals y Olson, 2004). En definitiva, es posible señalar que los estereotipos se caracterizan por ser el resultado de los procesos de categorización e interacción social, los cuales constituyen esquemas de la realidad que les permiten a las personas orientarse socialmente y adaptarse mejor al entorno (Tajfel, 1984).

Otro concepto asociado es el de metaestereotipo que hace referencia a las creencias que los miembros de un grupo concuerdan que otros grupos tienen sobre ellos. (Vorauer, Hunter, Main y Roy, 2000). En otros términos, se trata del "estereotipo del estereotipo" y ha despertado interés debido al impacto que tiene en las evaluaciones que realizan las personas tanto de los otros como de sí mismo (Kamans, Gordijn, Oldenhuis y Otten, 2009). Los autoestereotipos, conforme a lo anteriormente señalado, dicen relación con el conjunto de creencias y percepciones sobre si mismo y el grupo del cual se forma 
parte (Morales, Páez, Kornblit y Azún, 2002).

Los estereotipos pueden ser positivos 0 negativos Hewstone, Stroebe, Codol y Stepehnson, 1994), pero independiente de su tendencia, actúan como un proceso cognoscitivo y social que, utilizado como fuente de conocimiento, permite ahorrar esfuerzos de pensamiento en las relaciones interpersonales (Worchel, Cooper, Goethals y Olson, 2004) Si bien aquellas inferencias pueden conducir a juicios sociales precisos, los estereotipos también pueden inducir a ideas erróneas respecto de los otros, al centrarse solo en la información consistente con éste e ignorar aquellos elementos que lo contradicen. (Secord y Backman, 1976), lo anterior se acentúa si los estereotipos se desarrollan sin ninguna base en la realidad objetiva (Klineberg, 1973). Al sesgar el procesamiento de la información, los estereotipos pueden generar incluso, de manera inconsciente, distintos tipos de prejuicio y discriminación e influir en los roles, comportamientos y desempeño que tienen las personas en el medio social (Myers, 2004 y Kimble, Hirt, Diaz-Loving, Hosch, William y Zárate, 2002). Un claro ejemplo de ello, es el fenómeno conocido como "la amenaza del estereotipo" que da cuenta de cómo al conocer o creer que estamos frente a un estereotipo negativo sobre nuestro grupo, reaccionamos con estrés afectando negativamente nuestro desempeño (Steele y Aronson, 1995). En otras palabras, este fenómeno interfiere en el accionar de las personas al no querer confirmar el estereotipo negativo asociado a su categoría, constituyéndose así en un círculo vicioso que fomenta las profecías autocumplidas. (Worchel, Cooper, Goethals y Olson, 2004).

En virtud de la importancia de estos fenómenos en el comportamiento social, la presente investigación se centra en la descripción de los principales adjetivos que configuran el autoestereotipo y el metaestereotipo de los chilotes residentes en la comuna de Punta Arenas. De esta manera se busca no solo conocer su contenido sino también comparar la visión que tienen sobre sí mismos, con la que perciben que tiene el grupo dominante sobre ellos. La hipótesis de esta investigación entonces es que ambos, autoestereotipo y metaestereotipo, compartirán una parte importante de su contenido diferenciándose únicamente en algunos aspectos que resultan de particular importancia puesto que describen su visión de la realidad social.

Si bien el estudio es de carácter preliminar, puede constituir un primer acercamiento a una realidad regional nunca antes abordada desde el ámbito de la psicología social. Por tanto, la posibilidad de conocer elementos que forman parte de la identidad de la población chilota residente en la ciudad de Punta Arenas permitirá avanzar en la temática de cómo grupos de inmigrantes se relacionan con el medio social, dando paso a propuestas de intervención y acción por ejemplo desde el ámbito del Trabajo Social.

\section{MÉTODO}

\section{Participantes}

La muestra no probabilística de tipo intencional estuvo conformada por 65 personas (36 mujeres y 29 hombres) oriundas de Chiloé y/o descendientes de chilotes que actualmente viven en la comuna de Punta Arenas (el 96,9\% nació o vive más de 10 años en la región). En cuanto a descendencia indígena, un $64,6 \%$ de los encuestados manifestó no pertenecer a ningún pueblo originario de nuestro país y un $35,4 \%$ declaró tener descendencia indígena $(100 \%$ pueblo Mapuche Huilliche). Según edad, el $27,7 \%$ de la muestra posee 60 años o más, un 18,5\% posee entre 21 a 29 años, un 16,9\% pertenece al rango de 40 a 49 , un $15,4 \%$ al rango de 30 a 39 años y el mismo porcentaje al rango de 50 a 59 años de 
edad. Según actividad u oficio, un $32,3 \%$ resulta ser trabajador dependiente, un $18,5 \%$ trabajador independiente, un 12,3\% de los participantes son estudiantes y un $36,9 \%$ declara una actividad diferente a las ya mencionadas.

\section{Instrumentos}

Técnica de generación de adjetivos: El instrumento se compuso de 15 espacios para que los encuestados escribieran libremente las características típicas, según ellos, de los chilotes residentes en la comuna de Punta Arenas. Luego, mediante el mismo procedimiento, debían indicar los adjetivos típicos que le asignan otras personas a los chilotes (los magallánicos no chilotes). Posteriormente, se les pidió evaluar cada una de las características por ellos señaladas en su valencia utilizando una escala tipo Likert de 6 puntos (1: muy mala a 6: muy buena).

Técnica lista de adjetivos: Adaptado de Katz y Braly (1933). Se presentó una lista con 27 características con el fin de que los encuestados escojan 5 de ellas como las más típicas de los chilotes. Luego, las mismas características fueron evaluadas en su tipicidad por los chilotes según la percepción que tendrían los magallánicos no chilotes (metaestereotipo). Posteriormente, se les pidió evaluar cada una de las características por ellos señaladas en su valencia utilizando una escala tipo Likert de 6 puntos (1: muy mala a 6: muy buena). Dicha técnica se utilizó como indicador de validez para corroborar la uniformidad de los adjetivos asociados al autoestereotipo y metaestereotipo de los chilotes patagónicos.

Escala de identificación con el endogrupo: Compuesta por 4 afirmaciones que buscaban conocer el grado de acuerdo (escala tipo Likert, 1: para nada de acuerdo a 5: totalmente de acuerdo) de los encuestados con relación al orgullo que sienten por la historia de Chiloé, si consideran que Chiloé es el mejor lugar del mundo, si los valores de los chilotes debiesen ser un modelo a seguir y si provenir de Chiloé constituye un privilegio. Mostró una confiabilidad 0,83 (alfa de Cronbach).

Identificación con el endogrupo: Mediante un ítem "Qué tanto se identifica con su etnia", se evaluó el nivel de identificación con dicha categoría social (escala tipo Likert, 1: para nada a 5: totalmente).

\section{Procedimiento}

Los participantes fueron contactados uno a uno $\mathrm{e}$ invitados a responder los instrumentos. Cada colaborador recibió información respecto a la confidencialidad de sus respuestas y los objetivos generales del estudio, firmando un consentimiento informado. La aplicación fue autoadministrada, con un tiempo total promedio de 15 minutos. Al finalizar el cuestionario se contestó a sus preguntas y se les agradeció por su participación (Estrada, 2005).

\section{RESULTADOS}

Estereotipos mediante técnica de generación de adjetivos: Los adjetivos asignados al propio grupo (autoestereotipo) fueron reducidos utilizando la técnica de análisis de contenido mediante la repetición, sinonimia y significados afines (Cárdenas, 2006). De esta forma de las 126 expresiones asociadas al autoestereotipo, se construyeron 38 categorías, siendo las 10 más frecuentes consideradas para el análisis. Los resultados indican que la categoría más mencionada fue "trabajador" $(80 \%)$, seguida por "amable" $(55,4 \%)$, "generoso" (41,5\%) "esforzado" (41,5\%), "honesto" (29,2\%), "responsable" (24,6\%), "humilde" (18,5\%), "costumbrista" $(16,9 \%)$, "respetuoso" (15,4\%) e "inteligente" (13,8\%). Se aprecia que el $90 \%$ de las categorías es de tendencia positiva y el $10 \%$ resulta ser neutra (ver Tabla 1). 
Al evaluar la valencia del autoestereotipo, la muestra presentó un promedio alto que se sitúa en el polo positivo $(M=5,23, D E: 0,71)$ ya que existe una diferencia significativa con el punto neutro de la escala, $M=3,5, t_{(64)}=19,38, p=$ 0,0001 .

Siguiendo el mismo procedimiento del autoestereotipo, las 137 expresiones asociadas al metaestereotipo fueron reducidas a 42, de las cuales las 9 más frecuentes se sometieron a análisis. Los resultados indican que la categoría más mencionada fue "trabajador" (41,5\%), seguida por "amable" $(35,4 \%)$, "esforzado" $(24,6 \%)$, "generoso" $(24,6 \%)$, "ignorante" $(24,6 \%)$, "honesto" $(15,4 \%)$, "humilde" $(13,8 \%)$, "costumbrista" (12,3\%) y "mala persona" (12,3\%). Se aprecia que el 66,7\% de los metaestereotipos se refieren a características positivas, un $22,2 \%$ corresponde a características negativas y solo un $11,1 \%$ tiene tendencia neutra.

Al comparar el autoestereotipo y el metaestereotipo, vemos que se diferencianprincipalmente por 4 rasgos 0 adjetivos. Mientras al evaluar a su propio grupo este es caracterizado utilizando los términos "inteligente, respetuoso y responsable", el metaestereotipo contiene los términos "ignorante y mala persona". En otros palabras, la visión del propio grupo, utiliza tres adjetivos positivos que desaparecen al momento de dar cuenta de la visión que el otro grupo tiene sobre el propio y aparecen dos nuevos adjetivos negativos (ver Tabla 1).

Al evaluar la valencia del metaestereotipo, la muestra presentó un promedio sobre el punto neutro de la escala $(M=4,49, D E: 1,41)$ indicando positividad, $M=3,5, \mathrm{t}_{(64)}=5,66, p=0.0001 . \mathrm{Al}$ comparar la valencia asignada a los componentes del autoestereotipo y del metaestereotipo se observan diferencias significativas entre ambos que indican que el auto-estereotipo ( $M: 5,23, D E: 0,71)$ es más positivo que el metaestereotipo $(M$ : $4,49, D E: 1,41),\left(t_{c(64)}=4,37, p=0,0001\right)$.

TABLA 1.

Frecuencia y porcentaje de los adjetivos contenidos en el autoestereotipo y metaestereotipo mediante técnica de generación de adjetivos.

\begin{tabular}{|c|c|c|c|c|}
\hline \multirow{2}{*}{ Adjetivo } & \multicolumn{2}{|c|}{ Autoestereotipo } & \multicolumn{2}{|c|}{ Metaestereotipo } \\
\hline & Frecuencia & Porcentaje & Frecuencia & Porcentaje \\
\hline Amable & 36 & 55 & 23 & 35 \\
\hline Costumbrista & 11 & 17 & 8 & 12 \\
\hline Esforzado & 27 & 42 & 16 & 25 \\
\hline Generoso & 27 & 42 & 16 & 25 \\
\hline Honesto & 19 & 29 & 10 & 15 \\
\hline Humilde & 12 & 19 & 9 & 14 \\
\hline Inteligente & 9 & 14 & - & - \\
\hline Ignorante & - & - & 16 & 25 \\
\hline Mala persona & - & - & 8 & 12 \\
\hline Respetuoso & 10 & 15 & - & - \\
\hline Responsable & 16 & 25 & - & - \\
\hline Trabajador & 52 & 80 & 27 & 42 \\
\hline
\end{tabular}

- $\quad$ Adjetivo que no presenta una alta frecuencia 
Estereotipos mediante lista de adjetivos: El cuestionario poseía un total de 27 adjetivos ya dados, dentro de los cuales 11 mostraron mayor frecuencia conformando el autoestereotipo. Los resultados indican que la opción más mencionada fue "trabajador" $(95,4 \%)$, seguida por "sociable" $(55,4 \%)$, "responsable" $(46,2 \%)$, "generoso" $(43,1 \%)$, "honesto" (40,0\%), "educado" (36,9\%), "agradable" (33,8\%), "inteligente" $(29,2 \%)$, "creativo" (20,0\%), "calmado" (18,5\%) y tímido (18,5\%). Se aprecia que el $82 \%$ de las características es de tendencia positiva y $18,1 \%$ resulta ser neutra (ver Tabla $N^{0} 2$ ). Al evaluar la valencia del autoestereotipo se observa que está se sitúa en el polo positivo $(M=5,14, D E: 0,75)$, ya que existe una diferencia significativa con el punto neutro de la escala, $M=3,5, t_{(63)}=17,66, p=$ 0,0001 .

Al igual que para el caso de los adjetivos asignados al propio grupo (autoestereotipo) obtenidos mediante la técnica "lista de adjetivos", el cuestionario poseía un total de 27 características ya dadas, dentro de las cuales 13 mostraron mayor frecuencia conformando el metaestereotipo. Los resultados indican que el adjetivo más mencionado fue "trabajador" (78,5\%), seguido por "tímido" $(40,0 \%)$, "responsable" (38,5\%), "ignorante" (35,4\%), "sociable" (35,4\%), "generoso" (27,7\%), "educado" (24,6\%), "creativo" (23,1\%), "agradable" (21,5\%), "calmado" (21,5\%), "honesto" (16,9\%), "flojo" (15,4\%) e "inteligente" (15,4\%). Se aprecia que el $69,2 \%$ corresponde a una característica positiva, el $15,4 \%$ a una opción negativa y un $15,4 \%$ a una característica de tendencia neutra (Ver Tabla 2). Al evaluar la valencia del metaestereotipo, la media se sitúa igualmente en el polo positivo $(M=4,54$, $D E: 1,2), M=3,5, t_{(63)}=7,003, p=0,0001$.

TABLA 2.

Frecuencia y porcentaje autoestereotipos y metaestereotipos obtenidos mediante técnica de adjetivos

\begin{tabular}{lcccc}
\hline \multirow{2}{*}{ Adjetivo } & \multicolumn{2}{c}{ Autoestereotipo } & \multicolumn{2}{c}{ Metaestereotipo } \\
\cline { 2 - 5 } & Frecuencia & Porcentaje & Frecuencia & Porcentaje \\
\hline Agradable & 22 & 34 & 14 & 22 \\
Calmado & 12 & 19 & 14 & 22 \\
Creativo & 13 & 20 & 15 & 23 \\
Educado & 24 & 37 & 16 & 25 \\
Flojo & - & - & 10 & 15 \\
Generoso & 28 & 43 & 18 & 28 \\
Honesto & 26 & 40 & 11 & 17 \\
Ignorante & - & - & 23 & 35 \\
Inteligente & 19 & 29 & 10 & 15 \\
Responsable & 30 & 46 & 25 & 39 \\
Sociable & 36 & 55 & 23 & 35 \\
Tímido & 12 & 19 & 26 & 40 \\
Trabajador & 62 & 95 & 51 & 79 \\
\hline
\end{tabular}

- $\quad$ Adjetivo que no presenta una alta frecuencia 
Al comparar el auto y meta-estereotipo, se observa que el segundo está compuesto por dos características que no son elegidas para el auto-estereotipo, estos son "flojos e ignorantes". En otros términos, la visión que consideran compartida por otros grupos sobre el propio, incluye dos características negativas que no son utilizadas para autodescribirse.

Al comparar la valencia asignada a los componentes del autoestereotipo $(M=5,14$, $D E: 0,75)$, y del metaestereotipo $(M=4,54$, $D E: 1,2)$ obtenidos mediante la técnica "lista de adjetivos" se observan diferencias significativas entre ambos $\left(t_{c(63)}=3,81, p=\right.$ $0,0001)$ que indican que el auto-estereotipo es mejor evaluado que el meta-estereotipo.

Finalmente se realizó un análisis de distancias entre los adjetivos utilizados, con ambas técnicas para el auto y meta estereotipo con el fin de visualizar las diferencias en la organización interna de los mismos. Mediante el escalamiento multidimensional se produjo figuras en las que es posible observar que la forma en que se estructuran los adjetivos tienden a ser similares, y a lo menos una dimensión parece oponer el adjetivo "trabajador" con el de "inteligente". Al comparar los autoestereotipos, ambas técnicas producen los adjetivos "generoso", "honesto", "inteligente", "responsable" y "trabajador", mientras que se diferencian en los adjetivos "costumbrista" y "esforzado" generados en la técnica libre, como también "creativo" y "educado" asignados en la técnica de selección. Al comparar los metaestereotipos, ambas técnicas producen los adjetivos "generoso", "honesto", "ignorante" y "trabajador", diferenciándose en el adjetivo "mala persona" obtenido mediante la técnica abierta y las características "creativo" y "flojo" escogidas a través de la técnica de selección (Ver Figura 1). Con relación al potencial impacto diferencial de las variables sociodemográficas, nuestros resultados no presentan diferencias significativas en cuanto a los contenidos de los estereotipos.

\section{Identificación}

Con el grupo originario: Los resultados de la variable "identificación con su pueblo originario" indican que del total de participantes, un $60,9 \%$ se identifica con este grupo. Al medir el grado de identificación de aquellos que se reconocen como parte de un pueblo originario, encontramos un promedio alto $(M=4,21, D E: 1,12)$, ya que se sitúa sobre el punto medio o neutro de la escala, $M$ $=3,0, t_{(13)}=4,05, p=0,001$.

Con el grupo de chilotes: Los resultados indican que los participantes poseen un alto nivel de identificación con el grupo de chilotes, puesto que la media se sitúa en el polo positivo de la escala $(M=17,43, D E: 2,8), M=$ $12, t_{(63)}=15,8, p=0,0001$. Al separar a los participantes en dos grupos según su puntaje en identificación, se observa que aquellos más identificados con su endogrupo tienden a evaluar de manera más favorable el autoestereotipo $(M=5,4, D E: 0,51)$ que los que se reconocen en menor grado como chilotes $(M=4,9, D E: 0,83), t_{(62)}=-3,1, p<$ 0,003 . Lo mismo ocurre para el caso del metaestereotipo, el cual es evaluado de forma más positiva por los participantes que se sienten mayormente identificados con su endogrupo $(M=4,9, D E: 1,2)$ que por el resto de los chilotes que colaboraron en el estudio $(M=4,2, D E: 1,14), t_{(62)}=-2,4, p<0,020$. En otros términos, al comparar la valencia asignada al autoestereotipo y al metaestereotipo, en la información recogida por el método de "lista de adjetivos" según nivel de identificación, se observa que los más identificados con el grupo, presentan un promedio mayor que el de aquellos que no se sienten tan identificados con el pueblo chilote. Al realizar el mismo procedimiento con las valencias asignadas al autoestereotipo y metaestereotipo obtenidos mediante la técnica de "generación de adjetivos" se obtuvo un resultado que tiende a ser similar pero con diferencias no significativas. 
FIGURA 1.

Análisis de distancia entre adjetivos asignados al autoestereotipo y metaestereotipo utilizando la técnica generación de adjetivos y lista de adjetivos
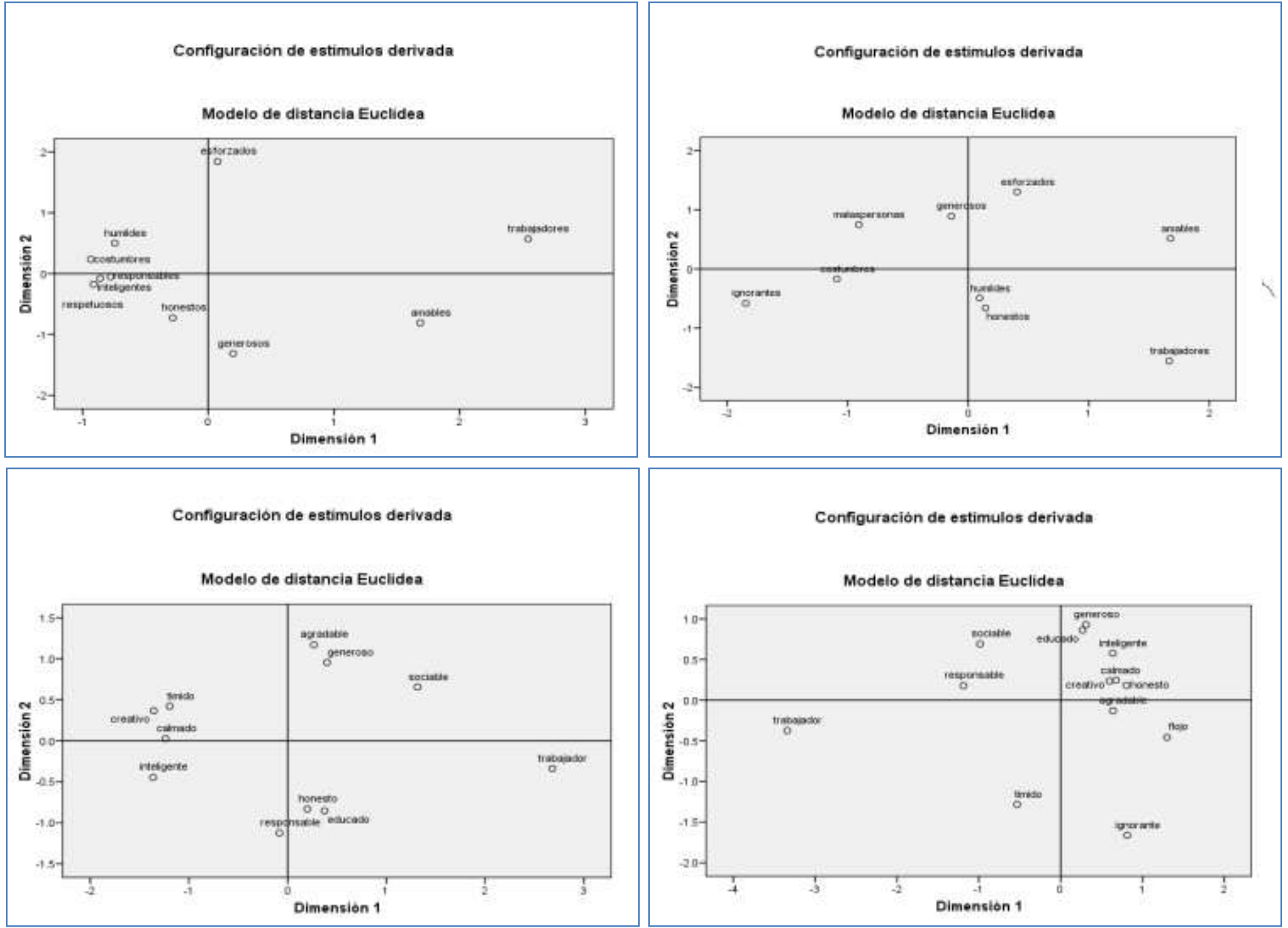

\begin{tabular}{l|l|l|l|l} 
SALUD \& SOCIEDAD | V. 2 №. 3 SEPTIEMBRE - DICIEMBRE & 2011
\end{tabular} 


\section{DISCUSIÓN Y CONCLUSIONES}

La presente investigación tuvo como propósito general describir los principales adjetivos que configuran aquellos aspectos de la identidad social ligados al autoestereotipo y metaestereotipo de un grupo de chilotes patagónicos, así como también conocer el nivel de identificación que manifiestan éstos con su endogrupo.

Los resultados obtenidos en los análisis, permiten concluir que los participantes poseen un autoconcepto considerablemente favorable. Lo anterior se manifiesta tanto en el contenido del autoestereotipo como también en la valencia que otorgan a estos adjetivos. El contenido del autoestereotipo incluye características de competencia como también de sociabilidad aunque se organizan dimensionalmente de forma diversa. Algunas de esas características son "trabajador", "generoso" y "honesto", las cuales se presentan no sólo en el autoestereotipo sino que también en el metaestereotipo.

No obstante, los resultados de la investigación indican que el autoestereotipo no coincide plenamente con el metaestereotipo, lo cual se visualiza en el hecho de que los participantes incluyen adjetivos negativos al momento de describir la forma en como son vistos por el exogrupo. A pesar de lo anterior, los elementos centrales del autoestereotipo se conservan, y la inclusión de algunos de valencia negativa configuran un metaestereotipo ambivalente que mantiene una tendencia a la valoración positiva. Lo anterior probablemente se debe a que los participantes tienen conciencia, 0 al menos la clara percepción de que el exogrupo (magallánicos no chilotes) no visualiza de forma completamente favorable a los chilotes, asignándoles características tales como "ignorantes", "flojos" y "malas personas". Esta tendencia a "negativizar" el metaestereotipo podría responder a la realidad (ser realmente evaluados de forma más negativa) 0 deberse a la tendencia a sobreestimar la negatividad de los estereotipos observada en otras investigaciones (Krueger, Sigelman y Tuch en Saiz, 2008). En definitiva, el hecho de que el autoestereotipo sea evaluado de manera más favorable que el metaestereotipo podría deberse a los procesos de sesgo endogrupal que tienden a favorecer o valorar mejor al propio grupo que al exogrupo (Tajfel, Billig, Bundy y Flament, 1971).

Desde una perspectiva metodológica, es posible afirmar que las dos técnicas utilizadas para el levantamiento de los estereotipos, es decir, la de generación de adjetivos y la de lista de adjetivos, producen resultados altamente similares. En consideración de lo anterior, la técnica "abierta", es decir, la de generación de adjetivos, produce una cantidad de información de mayor valor, que recoge aspectos inesperados, por lo que resulta una mejor opción para el estudio de los estereotipos.

Un resultado interesante fue el alto nivel de identificación mostrado por los participantes hacia su endogrupo. La pertenencia al pueblo chilote, parece proporcionarles una identidad social positiva que favorece la identificación con el grupo. Se trata de un grupo autovalorado positivamente y que además es percibido como una categoría social de alto valor para el exogrupo. Por lo tanto, puede suponerse que la estrategia de mantención de esta identidad positiva los impulsará a permanecer como miembros de éste y a buscar fortalecer esta identidad (Tajfel, 1984).

Sin duda, una de las fortalezas de esta investigación se sitúa en la doble aproximación de la medición del estereotipo en un grupo de chilotes patagónicos que no sólo aporta a la comprensión de la dinámica relacional en la comunidad de la ciudad de Punta Arenas, sino que permite afirmar que 
el contenido de los estereotipos evaluados se valida en similar producción obtenida tanto al definir espontáneamente adjetivos como al elegirlos de una lista propuesta. La naturaleza intencionada de la muestra como también su tamaño son las principales debilidades de nuestro estudio, ya que no permiten formular generalizaciones en base a los resultados obtenidos, por tanto resulta de interés para futuras investigaciones ampliar la muestra e incluso incorporar a otras comunas de la provincia con el fin de establecer correlaciones entre ellas.

Una de las posibles aplicaciones de los resultados obtenidos es que permiten conocer los contenidos de los estereotipos en vías de comprender las relaciones intergrupales que surgen en la relación "minoría - mayoría". En este sentido, futuras investigaciones deberían interesarse también en la postura de aquellos que se sitúan como el grupo mayoritario, es decir, los "magallánicos no chilotes". Además, resulta necesario conocer las consecuencias reales que a nivel de la convivencia regional pudiesen tener las características negativas asignadas al metaestereotipo de los chilotes, como es el caso del adjetivo "ignorantes" (35,4\% frecuencia mediante lista de adjetivos y $24,6 \%$ a través de la técnica generación de adjetivos) al vincularlos con escenarios referidos al empleo, la educación y otros. Sin duda, profundizar en estas materias podría contribuir a entender los procesos de relación intergrupal existente y generar propuestas de intervención/acción desde diversas disciplinas y con un claro nivel de pertinencia local.

\section{REFERENCIAS}

Banon, R. y Byme, D. (2005). Psicología Social. Madrid, España: Prentice Hall.

Cárdenas, M. (2006). El análisis multivariante de las representaciones sociales. Antofagasta: Editorial Universidad Católica del Norte.

Estrada, C. (2005) "Manual Operativo de Metodología Cuantitativa 1.3". Universidad de Magallanes

Estrada, C., y Avendaño, S. (2008). ¿Consideran los magallánicos que su cultura posee una esencia única?: estudio descriptivo de la aplicación de un instrumento de evaluación de esencialismo cultural a una muestra puntarenense. Magallania, 36, (2), 155-162.

Hewstone, M., Stroebe, W., Codol, J. y Stephenson, G. (1994). Introducción a la Psicología Social: Una perspectiva europea. Barcelona, España: Ariel Psicología.

I.N.E. Instituto Nacional de Estadística (2002). Estadísticas demográficas de Chile, Censo 2002. Santiago de Chile

Kamans, E., Gordijn, E., Oldenhuis, H., y Otten, S. (2009). What I think you see is what you get: Influence of prejudice on assimilation to negative meta-stereotypes among Dutch Moroccan teenagers. European Journal of Social Psychology, 39, 842-851

Katz, D., Braly, K. (1933). Racial stereotypes of one hundred students. Journal of abnormal and Social Psycology. 28, 280-290

Kimble, Ch. Hirt, E. Diaz-Loving, R. Hosch, H. William Lucker, G. \& Zárate, M. (2002). Psicología Social de las Américas. México: Pearson Educación.

Klineberg, Otto. (1973). Psicología Social. México: Fondo de cultura económica.

Larraín, J. (2001). Identidad Chilena. Santiago, Chile: Lom

Lippman (1922) Public Opinión Quarters. New Cork: The Free Press

Martinic, M. (2002). Breve historia de Magallanes. Punta Arenas, Chile: Universidad de Magallanes.

Morales F., Paez, D. Kornblit, A. y Azún, D. (2002). Psicología Social. (1 ${ }^{\text {a }}$, ed). Buenos Aires, Argentina: Prentice Hall

Myers, D. (2004). Exploraciones de la Psicología Social. Madrid, España: McGraw-Hill. 
Saiz, J., Merino, M., y Quilaqueo, D. (2009). Meta-estereotipos sobre los indígenas mapuches de Chile. Interdisciplinaria, 26, (1), 23-48.

Saiz, J., Rapiman, M.., y Mladinic, A. (2008). Estereotipos Sobre los Mapuches: Su Reciente Evolución. Psykhe, 17, (2), 27-40.

Sandoval, M. (1998) El Trabajo Social chileno en un contexto de mutación cultural: Desafíos para la acción y la formación. Revista de Trabajo Social Perspectivas, notas sobre intervención y acción social. 7 , 5-19.

Scandroglio, B., López Martínez, J., y San José Sebastián, M. (2008). La Teoría de la Identidad Social: una síntesis crítica de sus fundamentos, evidencias y controversias. Psicothema, 20, (1), 80-89.

Secord, P., Backman, C. (1976). Psicología Social. México: Mc Graw-Hill

Steele, C., y Aronson, J. (1995). Stereotype threat and the intellectual test performance of African Americans. Journal of Personality and Social Psychology, 69(5), 797-811.

Tajel, H. (1984). Grupos humanos y categorías sociales. Barcelona: Editorial Herdey

Tajfel, H., Billig, M., Bundy, R. y Flament, C. (1971). Social categorization and intergroup behaviour. European Journal of Social Psychology, 2, 149-178.

Tajfel, H. y Turner, J. (1979). An integrative theory of intergroup conflict. The social identity theory of intergroup behaviour. In W. G. Austin y S. Worchel (Eds.), The social psychology of intergroup relations. Monterey, CA: Brooks/Cole.

Therborn, G. (2000). Identidades Nacionales y otras identidades. Revista de Sociología Departamento de Sociología Universidad de Chile. 10, 139-152.

Vorauer, J. Hunter, A., Main, K. y Roy, S. (2000). Meta-Stereotype Activation: Evidence From Indirect Measures for Specific Evaluative Concerns Experienced by Members of Dominant Groups in Intergroup Interaction. Journal of Personality and Social Psychology, 78, (4), 690-707

Worchel, S., Cooper, J., Goethals, G. y Olson, J. (2004). Psicología Social. Madrid: Editorial Thomson. 